\title{
Management of Perioperative Anemia in Patients that Performed Abdominal Major Surgery
}

\author{
Majlinda Naço ${ }^{1,2, *}$, Haxhire Gani ${ }^{2}$, Vjollca Duro ${ }^{1,3}$, Eden Naço ${ }^{4}$
}

Received: 03 December 2021 / Accepted: 24 December 2021 / Published online: 20 January 2022

This article is published with open access at https://journal.astes.org.al

(C) The author(s) 2022. \& Copyright (C) 2022, the Albanian Society for Trauma and Emergency Surgery

(c) The Albanian Journal of Trauma and Emergency Surgery is an Open Access Journal. All articles are distributed under the terms of the Creative Commons Attribution Non-Commercial License: http://creativecommons.org/licenses/by-nc/4.0/) which permits unrestricted non-commercial use, distribution, and reproduction in any medium provided the original work is properly cited.

\begin{abstract}
Introduction: Anemia is a recognized predictor of adverse postoperative outcomes in patients during abdominal major surgery. The management of perioperative anemia involves many strategies. Pre-operative anemia has adverse outcomes in major surgery and is called the fourth factor. Anemia was then classified into mild, moderate, and severe depending on hemoglobin level. It is present almost in $40 \%$ of patients that performed elective major surgery. Patients with preoperative anemia are associated with an increased rate of blood transfusion together with a risk for high morbidity and mortality. The most usual cause of preoperative anemia is iron deficiency, which can be treated with oral/intravenous iron depending on the time scheduled for surgery. A review concluded that anemia recovery earlier with preoperative intravenous iron than with oral iron supplement. A perioperative erythropoietin injection is also a reasonable approach for patients with hemoglobin between 10 and $13 \mathrm{~g} / \mathrm{dL}$ and if autologous blood donation is performed. The protocol requires a baseline complete blood count and iron studies and all patients should receive iron supplementation during erythropoietin therapy. The next strategy is stabilized macro/ microcirculation to optimize the patient's tolerance to bleeding. Recent recommendation, in general, suggests no bridging therapy consider it only if high thrombotic risk. Finally, targeted surgery should be used to reduce intraoperative and postoperative bleeding. We recommend a restrictive transfusion strategy. Allogeneic blood transfusion is associated with an increased incidence of nosocomial infections. Postoperative anemia must be treated with the use of intravenous iron. We ought to know fresh frozen plasma used in deficit in factor V, XI, in dose $15-30 \mathrm{ml} / \mathrm{kg}$ and be aware of its complications as immunomodulation, acute lung injury, and cardiac overload.
\end{abstract}

Conclusion: Our goal is to improve patients' clinical outcomes.

Keywords: anemia, blood, blood plasma, iron deficiency anemia, transfusion-transmitted infections.

\section{Introduction}

The objective of these papers is to provide the anesthetists and the surgeons with an overview of the perioperative anemia how to improved really clinical management of patients. As we know, the anemia in the abdominal surgical

Original article, no submission or publication in advance or in parallel

* Corresponding author:

Asc. Prof. Dr. Majlinda Naço, MD, Ph.D

$\bowtie$ majlinda_naco@yahoo.com

1 University of Medicine of Tirana, ALBANIA

2 Service of Anesthesia and Intensive Care in General Surgery, UHC "Mother Theresa", Tirana, ALBANIA

3 Department of Microbiologist, UHC "Mother Theresa", Tirana, ALBANIA

4 Medical student at Catholic University "Our lady of good Counsel", Tirana, ALBANIA. patients has a significant impact on perioperative morbidity and mortality. Anemia is a health problem and is called the fourth factor that has to do with very low red blood cells or low hemoglobin. Hemoglobin is an iron-rich protein that binds to oxygen in the lungs and transports it to every tissue and cell. Global anemia rates are very high. According to Word Health Organization a patient is anemic when the level of hemoglobin is under $13 \mathrm{gdl}^{-1}$ in men, hemoglobin is $<12 \mathrm{gdl}^{-1}$ in women and when the hemoglobin is $<11 \mathrm{gdl}^{-1}$ in pregnant [1]. Anemia was then classified into mild when the level of hemoglobin $<12 \mathrm{gr} / \mathrm{dl}^{-}{ }^{1}$, moderate the level of hemoglobin $<10 \mathrm{gr} / \mathrm{dl}^{-1}$, and severe the level of hemoglobin $<7 \mathrm{gr} / \mathrm{dl}^{-1}$, depending of hemoglobin level.

On the basis of the level of hemoglobin anemia is divided into first grade anemia $11.5 \mathrm{gr} / \mathrm{dl}^{-1}$ : the level of hemoglobin is under $10 \mathrm{gr} / \mathrm{dl}^{-1}$ : is called second grade anemia (9-7 gr $/ \mathrm{dl}^{-1}$ ) and deep anemia (third grade) when the level of hemoglobin is less than $7 \mathrm{gr} / \mathrm{dl}^{-}$.

Another reference: Third degree anemia the level of hemoglobin 6.5-8 $\mathrm{gr} / \mathrm{dl}^{-1}$ and fourth degree the level of 
hemoglobin $<6.5 \mathrm{gr} / \mathrm{dl}^{-1}$ [2].

In an European study estimated the prevalence of preoperative anemia to be $31.1 \%$ in women and $26.5 \%$ in men. Anemia is associated with difficult outcome and need of blood transfusion especially during the SARS-CoV-2 pandemic that multiply the need for Perioperative Bleeding Management implementation [3]. Anemic patients very often presented to surgery without considered assessment and management of this risk factor for adverse clinical outcome [4]. The presence of anemia should be investigated in all major abdominal surgery with expected blood loss $(>500 \mathrm{ml}$ ). When treating anemia pre-operatively, the target hemoglobin concentration should be $\geq 13 \mathrm{gr} / \mathrm{dl}^{-}{ }^{1}$, in both sexes, to minimize the risk of transfusion associated different clinical outcomes [14].

There are a number of guidelines from professional associations recommending "what to do".

In 2013, the European Society of Anesthesia realized guidelines for the management of severe perioperative bleeding and first update in 2016 with the additional information to assist the doctors to Prepare, Plan and take Action and made these guidelines the first on patient blood management that does it means "how to do". The Patient blood management is a wide program aimed at minimizing the need for red cell transfusion and thus improving postoperative clinical outcome and reduced health care costs $[4,5]$.

\section{Prepare}

Prepare for any potential bleeding risks by performing preoperative assessments, particularly to detect anemia and allow time for its correction. Perioperative anemia increases the risk of numerous complications. Preoperative anemia has been shown to be a strong predictor for perioperative transfusion of allogeneic blood products such as Red Blood Cells, which itself carries a significant risk of different events and mortality [11]. Various strategies for the correction of pre and postoperative anemia have evolved. The implementation of a patient blood management program, which included patient assessment 4 weeks before surgery, was shown to be effective in reducing the rate of preoperative anemia [12]. Other groups have successfully used Patient Blood Management program ought to assess for anemia about 3-8 weeks before surgery. Assessment of patients 3 to 8 weeks before elective surgery provides enough time to initiate treatment and to take effect. This recommendation is also in agreement with current consensus and practical recommendations [5]. We need to identifying the cause (iron deficiency, renal insufficiency or inflammation). Anemia related to iron deficiency is common. Iron is the most common and widespread nutritional deficiency, even in industrialized countries, and affects about two billion people worldwide $[8,9]$. Iron deficiency, with or without anemia, is associated with chronic conditions such as cancer (43\% across malignances), inflammatory bowel disease (45\%) [10].
Patient Blood Management program focus on three main "pillars" (first, optimization of hemopoiesis, through the interventions to increase hemoglobin concentration; second, minimizing blood loss and bleeding; and third, protect and optimize physiological tolerance of anemia) all of which have a preoperative, intraoperative and postoperative component [4]. Patient blood management has been shown to improve outcomes and reduce healthcare costs in a number of studies [4, 5]. Recent work shows how this systematic approach to optimizing hemoglobin in surgical patients has been effectively with improved 90day postoperative outcomes in a United Kingdom center [13]. Anemia in surgical patients is a common and serious problem; around $40 \%$ of patients presenting for intermediate or major surgery are anemic [14]. Anemia is a recognized predictor of adverse postoperative clinical postoperative morbidity and mortality. All patients for elective surgery in whom blood loss is expected to be $>500 \mathrm{ml}$ should have their hemoglobin checked pre-operatively and be investigated if they are found to be anemic. A recent systematic review found that anemic patients with rectal cancer have a worse overall survival and disease-free survival after receiving transfusion of blood. [15]. Transfusion of red cells is associated with increased all-cause mortality and cancerrelated mortality [16] as well as increased recurrence of colorectal cancer [17]. Whether the relationship between anemia, red cell transfusions and morbidity/mortality is the cause is unknown. Detection of preoperative anemia should be carried out as soon as possible, at least 14 days before elective surgery [18] and preferably more than 30 days before surgery [21].

Many studies have shown an increase in hemoglobin and a decrease in red cell transfusion when iron deficiency anemia is treated with iron therapy [18]. Iron deficiency anemia is the most common type of anemia both worldwide [19] and in the surgical population [20]. If anemia (hemoglobin $<130 \mathrm{gr} / \mathrm{dl}^{-}$) is suggested using point-ofcare testing, laboratory testing should follow; further evaluation should include full blood count, serum ferritin, transferrin saturation, vitamin B12 and folate, a marker of inflammation (e.g., serum C-reactive protein) and a marker of renal function (e.g., serum creatinine) [5]. The most usual cause of preoperative anemia is iron deficiency, which can be treated with oral/intravenous iron depends of the time scheduled for surgery. A review concluded that anemia recovery earlier with preoperative intravenous iron than with oral iron supplement [21]. Oral iron supplementation may be suitable for a lot of patients, and any side-effects are usually mild [2, 3, 4]. When the time between consultation and surgery is sufficient, oral iron treatment may be considered. Oral iron replacement should be targeted to patients with iron deficiency with or without anemia whose surgery is scheduled 6-8 weeks after diagnosis, preferably by the primary general physician. Daily $(40-60 \mathrm{mg})$ or alternate-day (80-100 mg) treatment with oral iron and nutritional advice should be initiated immediately in patients with iron deficiency and no contra-indications [5]. Oral 
iron is cheap and both oral and intravenous iron increase hemoglobin concentration, whereas diminish the need for red cell transfusion. According to literature it seems that oral iron increase hemoglobin $\sim 5,15 \mathrm{~g} \mathrm{dl}^{-1}$ within 30 days therapy [5].

At that large period of treatment is required for oral iron more side-effects occur. Gastro-intestinal side effects might lead to poor compliance with oral iron treatment. In our ward, monitoring of efficacy for oral iron is recommended after 4 weeks of treatment. A systematic review concluded that patients with preoperative iron deficiency anemia may have an earlier and more robust recovery of hemoglobin concentration with preoperative intravenous iron than with oral iron supplementation [5, 14, 24].

Intravenous iron should be used as front-line therapy in patients who do not respond to oral iron indicated if oral iron is poorly tolerated from the patient, is ineffective (no increase in hemoglobin after 4 weeks), if the major abdominal surgery is scheduled for $<6$ weeks after the diagnosis of iron deficiency, or in case of functional iron deficiency [2, 5, 24]. Single dose of intravenous iron seems to increase hemoglobin $>{\mathrm{hb} \sim 1.8 \mathrm{~g} \mathrm{dl}^{-}}^{-1}$. Intravenous iron is recommended by many clinical guidelines based largely on its effectiveness in reducing anemia. However, the association with important safety outcomes, such as infection, remains uncertain [22]. Iron supplement dosage is based on a formula used to calculate total iron deficiency [iron deficiency $=($ target $\mathrm{Hb}-$ actual $\mathrm{Hb}) \times 2.4 \times \mathrm{kg}$ weight +500 ] and on the cause of chronic iron loss [24]. Adjuvant therapy with erythropoiesis stimulating agents such as erythropoietin improves the effectiveness of iron therapy and reduces dose requirements. Actuality has shown that intravenous iron with or without adjuvant erythropoietin can reduce the need for transfusion in surgical patients. Perioperative erythropoietin injection is also a reasonable approach for patients with a hemoglobin between 10 and 13 $\mathrm{g} / \mathrm{dL}$ and if autologous blood donation is performed. The protocol requires a baseline complete blood count and iron studies and all patients should receive iron supplementation during erythropoietin therapy [Figure nrl and $\mathrm{nr}$ 2] [14,

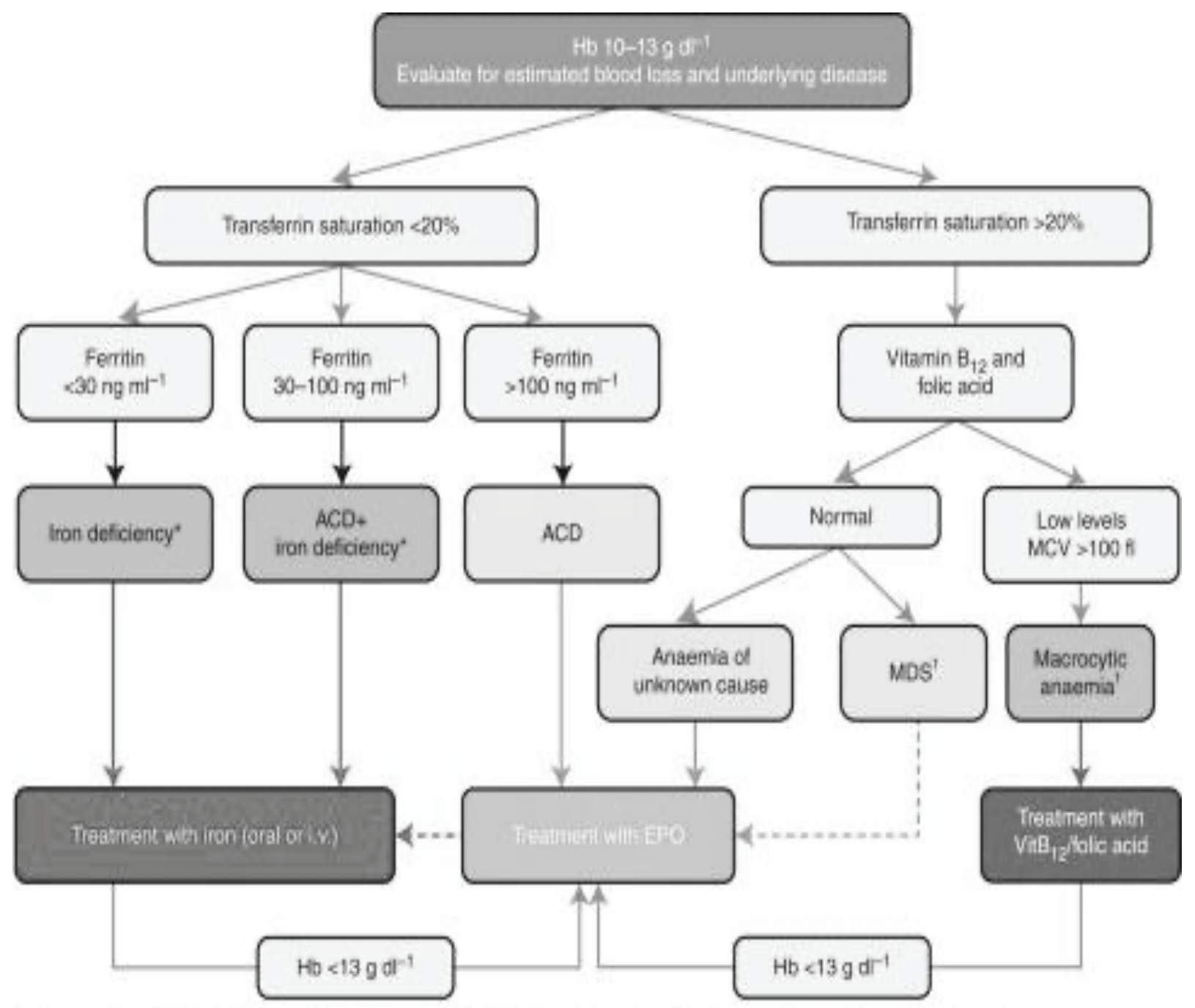

ACD, anaema of cheonic dsease: EPO orthyropoietin: MCV moan corpuscular volume; MDS, rmyeladysplaste syndrome.

Figure 1 Protocol of patient blood management 


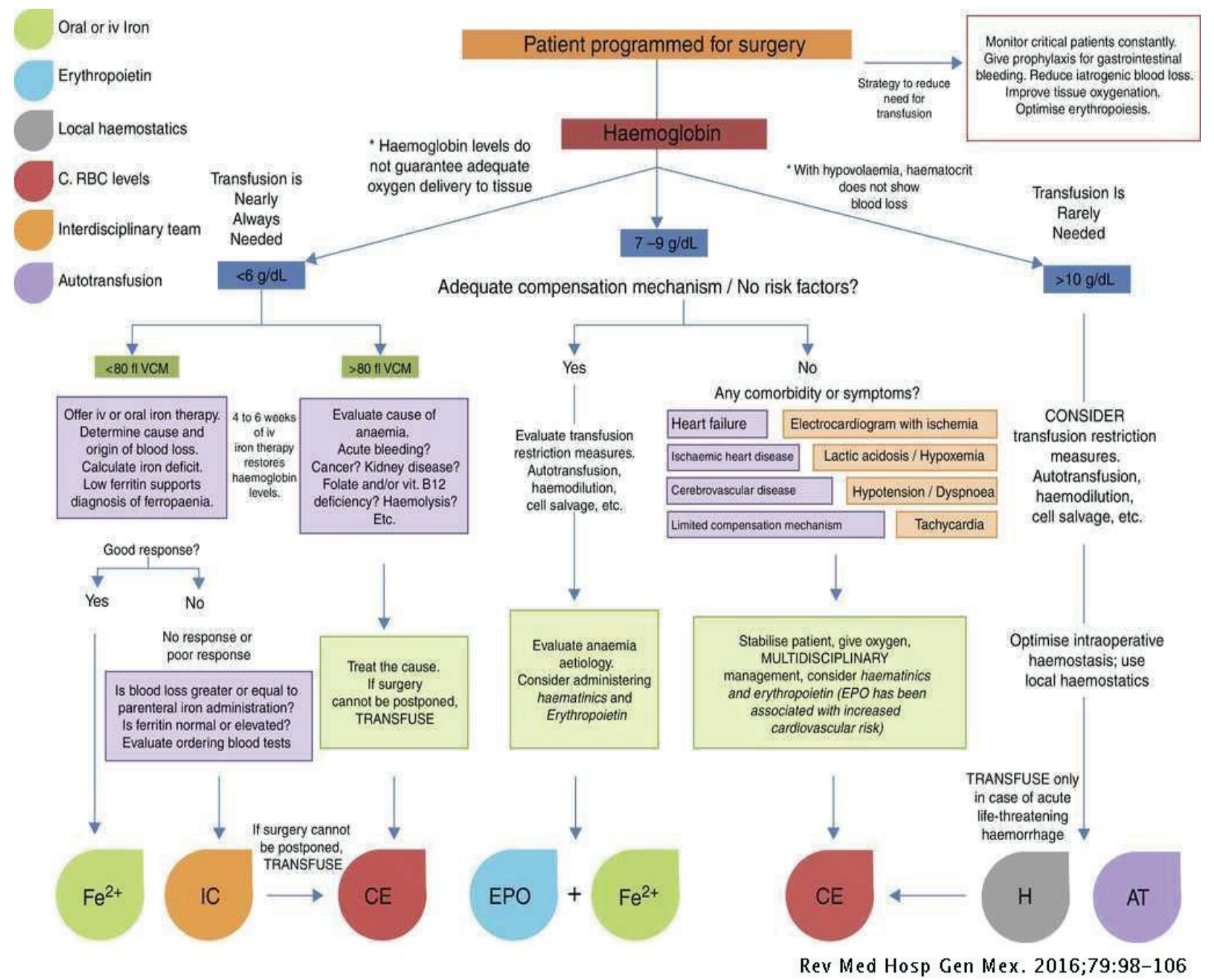

Figure 2 Algorithm for patient blood management on surgical ward

24]. Patients should receive erythropoietin injections on preoperative days 21, 14, and 7. Their last dose of erythropoietin should be on the day of the surgery $[4,5]$. However, chronic use of erythropoietin is associated with thrombotic cardiovascular events in cardiac and/or renal failure patients $[25,26)$.

A documented clinical history and totally physical examination are needed to determine potential risk factors for decompensation, bleeding or thrombosis. Particular attention should be paid to the patient's personal and family history. The European Society of Anesthesiology (ESA) strongly supports the development of high-quality, evidencebased clinical practice guidelines to help standardize the approach to patient care and to improve overall clinical practice.[27]

ESArecommends the use of standardized questionnaires on bleeding and drug history as preferable to the routine use of conventional coagulation screening tests such as activated partial thromboplastin time (APTT), international normalized ratio (INR) and platelet count in elective surgery. For the medications that affect hemostasis ESA recommends that aspirin therapy should continue perioperatively in most surgical settings. Where aspirin withdrawal before surgery is considered, ESA recommends a time interval of 3 days for all procedures where there is a very high bleeding risk. For intraoperative or postoperative bleeding clearly related to aspirin, it suggests that platelet transfusion be considered [5]. Minimum preoperative withdrawal for clopidogrel or prasugrel is 5 days in hemorrhagic risk for surgical patient while in high hemorrhagic risk for surgical patient will stop 7 days before. It suggests that the first postoperative dose of clopidogrel or prasugrel should be given no later than $24 \mathrm{~h}$ after skin closure.

We also suggest that this first dose should not be a loading dose. ESA recommends assessment of creatinine clearance in patients receiving direct oral anticoagulants (DOACs) who are scheduled for surgery. For intermediate and high bleeding risk procedures it recommends that rivaroxaban, apixaban and edoxaban should not be given for 2 days before the procedure (i.e., last oral intake 3 days before), pending a creatinine clearance (Cockcroft-Gault formula) above $30 \mathrm{ml}$ min_1 till 4 days before the procedure (i.e., last oral intake 5 days if the creatinine clearance is between 30 - and 50$\mathrm{ml}$ min_1). Recent recommendation suggests no bridging 
therapy. [5] According to Vitamin K antagonists (VKAs) ESA recommends that for low or moderate thrombotic risk patients [e.g., atrial fibrillation patients with CHADS2 score 4; patients treated for $>3$ months for a nonrecurrent venous thromboembolism (VTE)] undergoing procedures requiring INR less than 1.5, VKA should be stopped 3 to 5 days before surgery (acenocoumarin, warfarin). No bridging therapy is needed. Measure INR on the day before surgery and give $5 \mathrm{mg}$ oral vitamin $\mathrm{K}$ if INR exceeds 1.5. ESA recommends bridging therapy for high thrombotic risk patients (e.g., atrial fibrillation patients with a CHADS2 score $>4$; patients with recurrent VTE treated for less than 3 months; patients with a prosthetic cardiac valve). Warfarin: last dose 5 days before surgery; 4 days before surgery, no heparin; 3, 2 and 1 day before surgery, LMWH (last dose $24 \mathrm{~h}$ before surgery) SC daily; day 0, surgery. Acenocoumarin: 3 days before surgery, last dose; 2 and 1 day before surgery, same protocol as for warfarin. ESA suggest that the therapeutic dose of LMWH should be tailored for each patient, depending on the respective thrombotic and bleeding risks [5].

\section{Plan}

Plan for any intraoperative bleeding that may occur by using transfusion algorithms that incorporate pre-defined transfusion triggers to help guide hemostatic intervention, by being aware of the limitations of standard coagulation tests and by modifying the approach accordingly to use point-of care testing and others [5]. Perioperative bleeding management is a complex and changing field requiring multiple assessments and appropriate strategies to optimize patient care. There is an ongoing drive to find new alternatives to transfusion, a desire to reduce unnecessary use of blood products and a focus towards more evidencebased perioperative practice. Transfusion of RBCs is required only when the hemoglobin concentration decreases to levels at which overall nutrient demands cannot be met. The current mainstay of management for anemia in the older surgical patient, allogenic blood transfusion, can contribute to negative outcomes and is increasingly avoided where possible. Physicians are often asked to advise on

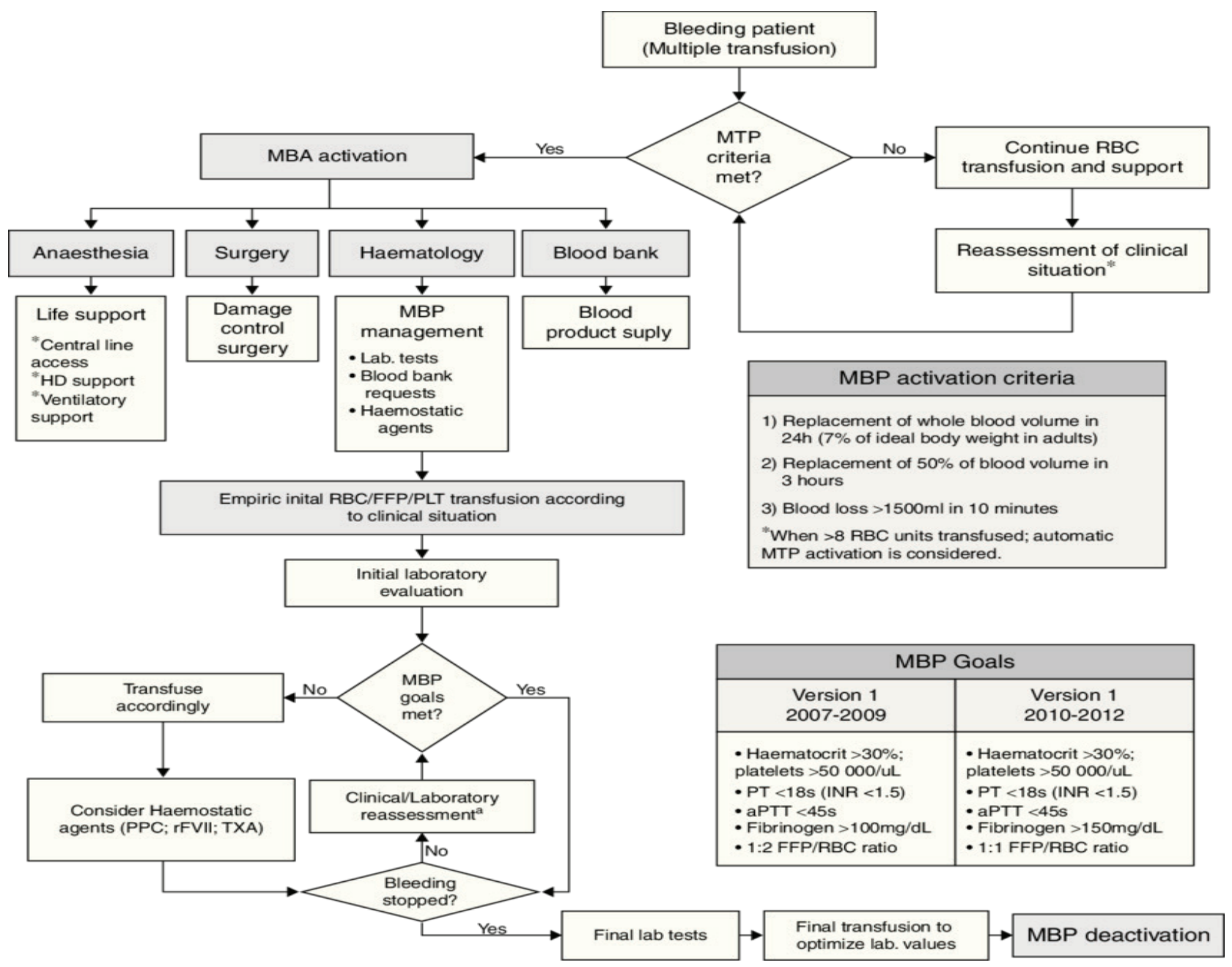

Figure 3. Massive transfusion protocol (MTP) algorithm. The boxes show the MTP activation criteria and MTP goals, used within the algorithm. RBC: red blood cells; FFP: fresh frozen plasma; PLT: platelet units; ICU: intensive-care unit; PT: prothrombin time; aPTT: activated partial thromboplastin time; PPC: prothrombin complex concentrate; rFVII: recombinant factor VII; TXA: tranexamic acid. 
optimizing anemic patients preoperatively. The advice provided varies widely between physicians and anesthetists or surgeons managing the same patient group. All those involved in preoperative optimization and postoperative management of older surgical patients should ensure that a considered and consistent approach to anemia is part of routine management. Allogeneic transfusion is emerging as a risk factor for many common complications in transfused patients, such as pulmonary lesion and hospital-acquired infection, and is a predictor of morbidity and mortality in critical patients. As a result, the notion of blood transfusion as a revitalizing treatment capable of improving a patient's overall status and speeding up recovery has been replaced by the more rational view that use other, less risky, methods must be used [4-10].

\section{Action}

If potential bleeding risks are known in advance and a plan of treatment is in place, the necessary Action can be set in motion as required [5]. In elective procedures with high risk of blood loss and need for transfusion, preoperative autologous donation can be considered. Elective surgery offers the potential for preoperative optimization of hemoglobin levels before undergoing surgery. In this procedure, a few units of the patient's blood are collected when is possible before surgery and reinfused to the patient perioperatively if needed. Patient blood management strategies during surgery generally focus on minimizing blood loss and improving tolerance of anemia. Vital signs should be closely monitored, and unnecessary hypovolemia and tachycardia should be avoided. Various options are available to limit blood flow to the site of surgery, thereby limiting blood loss: improving intraoperative hemostasis at the site of bleeding are: electrocautery and argon-beam coagulation, for cleaner cuts together with more effective hemostasis; topical hemostatic agents containing a mixture of thrombin, fibrinogen, calcium, collagen, gelatin, and cellulose act as a fast acting tamponade patient positioning to elevate the site of surgery, use of tourniquet, embolization or infusion of local vasoconstrictive agents. Hypotensive anesthesia is another approach to limiting blood loss, although hypotension must be closely monitored by a team of experienced anesthetists to ensure adequate perfusion of vital organs.

Another systemic approach to minimizing blood loss is to avoid hypothermia as hypothermia can adversely affect platelet function [24]. Surgery in cancer patients is challenging in both medical and transfusion terms. Surgery is usually more invasive, the surgical site is usually highly vascularized, and the clinical characteristics of the patients are far from ideal (preexisting anemia, advanced age, malnutrition, comorbidities, etc.). Because of this, indications for transfusion in these patients are less clear, and rely more on the experience of the surgeon than on guidelines. Massive bleeding affects delivery of blood to organs and tissues (due to hypovolemia), as well as the oxygen carrying capacity of blood (due to anemia) [5]. For any intraoperative bleeding first of all we need to utilizing transfusion algorithms. Transfuse of RBCs performed only when is really necessary, is required only when the hemoglobin concentration decreases to levels $<7 \mathrm{~g} \mathrm{dl}^{-}$. ESA recommends a restrictive transfusion strategy which is beneficial in reducing exposure to allogeneic blood products [5]. In these cases, fresh whole blood is essential for oxygen transport and adequate coagulation, since studies have shown that storing whole blood interferes with the metabolism, biochemistry, morphology and activity of red blood cells, and therefore affects the quality of the blood. It has also been reported that the longer the blood is stored, the greater the detrimental effect on coagulation [5, 24].

Although contamination of blood components with infectious agents represents a continuing challenge in transfusion medicine, rates of infection with known blood transmitted pathogens (e.g., HIV, HBV, HCV) are low following the implementation of high sensitivity testing methods. However, (re-) emerging pathogens remain a concern. Leukodepletion refers to the process of removing white blood cells from a unit of RBCs or platelets to a standardized degree. This is accomplished through either removal of the buffy coat following centrifugation or pre-storage filtration. The current consensus is that leukodepletion has defined indications in the prevention of three complications of blood transfusion: febrile non hemolytic transfusion reactions (HTRs), platelet refractoriness due to alloimmunization to human leukocyte antigen and transmission of cytomegalovirus. Leukodepletion process has been shown to be effective clinically and in cost too [29]. Clinically, leukodepletion might reduce the transmission of Creutzfeldt-Jacob disease and on the basis of accumulating evidence of leukocytemediated transfusion-related immunomodulation [30]. ESA recommends early and targeted treatment of coagulation factor deficiencies in the plasma. Sources of coagulation factors are coagulation factor concentrates, cryoprecipitate or high volumes of plasma, depending on the clinical situation, type of bleeding, type of deficiency and resources provide. Anesthetist and surgeon ought to know that fresh frozen plasma used in deficit in factor V, XI, in dose 15$30 \mathrm{ml} / \mathrm{kg}$ and always be aware of its complications as immunomodulation, acute lung injury and cardiac overload.

Finally targeted surgery should be used to reduced intraoperative and postoperative bleeding.

\section{Conclusions}

Management of perioperative anemia in patients that performed abdominal major surgery presents different challenges. Despite these challenges, evidence is emerging for methods of "transfusion sparing" intervention in order to optimize patients in the perioperative period. Given the current literature, we recommend employing patient blood 
management programs incorporating the ESA guidelines when assessing patients prior to elective surgery and the use of intravenous iron possibly with the addition of erythropoietin in the management of anemic abdominal surgical patients.

Such interventions could be employed using guidance from the PBM approach. More research is required into the methods of optimization in the anemic surgical patient, in order to provide definitive answers. Perioperative bleeding management is a wide concept that requiring multiple data and appropriate ways to realize patient care. Patient blood management is a program to the individual patient aimed at minimizing the donor for red cell transfusion and thus improving postoperative outcomes [3, 5]. The diagnosis and treatment of anemia and iron deficiency should commence as early as possible in the peri-operative period, and ideally as soon as the decision to undertake surgery is made. Major abdominal surgery should not be postponed to allow the diagnosis and treatment of anemia and iron deficiency. Sufficient data exist to support intravenous iron as efficacious and safe.

Many strategies to improve transfusion safety are recommended. The useful strategy is stabilized macro/ microcirculation that optimize the patient's tolerance to bleeding. ESA recommends a restrictive transfusion strategy which is beneficial in reducing exposure to allogeneic blood products. It recommends pathogen inactivation for fresh frozen plasma (FFP) and platelets, that labile blood components used for transfusion are leukodepleted.

ESA recommends that blood services implement standard operating procedures for patient identification and that staff be trained in early recognition of, and prompt response to, transfusion reactions. Our goal is to improve patients' clinical outcome and to reduce the mortality.

COI Statement: This paper has not been submitted in parallel. It has not been presented fully or partially at a meeting or podium or congress. It has not been published nor submitted for consideration beforehand. All authors declare that there is no conflict of interest.

This research received no specific grant from any funding agency in the public, commercial, or nonprofit sectors. There are no relevant or minor financial relationships from authors, their relatives or next of kin with external companies.

Disclosure: The authors declared no conflict of interest. No funding was received for this study.

Acknowledgement: We would like to thank medical staff of Service of Anesthesia and Intensive Care in General Surgery, UHC "Mother Theresa", Tirana, ALBANIA.

\section{References:}

1. World Health Organization. Nutritional Anemia: Report of a WHO Scientific Group. Geneva: WHO, 1968.

2. S. Lako, V. Ndreu, A Deda. Anemia ferriprive 34. https:// sites.google.com/a/infermieria.info/home/home/revista

3. Steinbicker A, Zurheiden NJ, Buckmann A, et al. Patient blood management: Umsetzung im Rahmen der Anaesthesiesprechstunde.Anaesth Intensivmed 2015; 56:64 74.

4. J. Partridge, D. Harari, J. Gossage, J. Dhesi. Anaemia in the older surgical patient: a review of prevalence, causes, implications and management. R Soc Med 2013: 106: 269277. DOI: $10.1177 / 0141076813479580$.

5. Sibylle A. Kozek-Langenecker, Aamer B. Ahmed, Arash Afshari\& al. Management of severe perioperative bleeding: guidelines from the European Society of Anaesthesiology Eur J Anaesthesiol 2017; 34:332-395.

6. Froessler B, Palm P, Weber I, \& al. The important role for intravenous iron in perioperative patient blood management in major abdominal surgery: a randomized controlled trial. Annals of Surgery 2016; 264: 41-6.

7. Akshay A. Shah, MSc; Killian Donovan, BM, BCh; Claire Seeley, \& al. Risk of Infection Associated with Administration of Intravenous Iron A Systematic Review and Meta-analysis. JAMA Network Open. 2021;4(11): e2133935. doi:10.1001/ jamanetworkopen.2021.33935.

8. WHO. Iron Deficiency Anaemia: Assessment, Prevention, and Control. A Guide for Programme Managers. Geneva: WHO, 2001.

9. Pasricha SR, Drakesmith H, Black J, Hipgrave D, Biggs BA. Control of iron deficiency anemia in low- and middle-income countries. Blood 2013; 121: 2607-17.

10. Peyrin-Biroulet L, Williet N, Cacoub P. Guidelines on the diagnosis and treatment of iron deficiency across indications: a systematic review. American Journal of Clinical Nutrition 2015; 102: 1585-94

11. Schiergens TS, Rentsch M, Kasparek MS, et al. Impact of perioperative allogeneic red blood cell transfusion on recurrence and overall survival after resection of colorectal liver metastases. Dis Colon Rectum 2015; 58:74-82.

12. Theusinger OM, Kind SL, Seifert B, et al. Patient blood management in orthopaedic surgery: a four-year follow-up of transfusion requirements and blood loss from 2008 to 2011 at the Balgrist University Hospital in Zurich, Switzerland. Blood Transfus 2014; 12:195-203.

13. Kotze A, Carter LA, Scally AJ. Effect of a patient blood management programme on preoperative anaemia, transfusion rate, and outcome after primary hip or knee arthroplasty: a quality improvement cycle. Br J Anaesth 2012; 108: 943-52.

14. K. E. Munting,A. A. Klein Optimisation of pre-operative anaemia in patients before elective major surgery - why, who, when and how? https://doi.org/10.1111/anae.14466 First published: 02 January 2019 Anaesthesia 2019, 74 (Suppl. 1), 49-57. 
15. Wilson MJ, van Haaren M, Harlaar JJ, et al. Long-term prognostic value of preoperative anemia in patients with colorectal cancer: a systematic review and meta-analysis. Surgical Oncology 2017; 26: 96-104.

16. Acheson AG, Brookes MJ, Spahn DR. Effects of allogeneic red blood cell transfusions on clinical outcomes in patients undergoing colorectal cancer surgery: a systematic review and meta-analysis. Annals of Surgery 2012; 256: 235-44.

17. Amato A, Pescatori M. Perioperative blood transfusions for the recurrence of colorectal cancer. Cochrane Database of Cochrane Database of Systematic Reviews 2006; 1: CD005033.

18. National Institute for Health and Clinical Excellence. Blood transfusion. Quality standard [QS138]. 2016. https://www. nice.org.uk/guidance/qs138 (accessed 22/03/2018)

19. Iron deficiency anemia: assessment, prevention and control. A guide for programme managers. 2001. http://www.who. int/nutrition/publications/micronutrients/anaemia_iron deficiency/WHO_NHD $01.3 / \mathrm{en} /$ (accessed 22/03/2018).

20. Shander A, Knight K, Thurer R, \& al.Prevalence and outcomes of anemia in surgery: a systematic review of the literature. The American Journal of Medicine 2004; 116(Suppl 7A): 58S-69S.

21. Goodnough LT, Maniatis A, Earnshaw P, et al. Detection, evaluation, and management of preoperative anaemia in the elective orthopaedic surgical patient: NATA guidelines. British Journal of Anaesthesia 2011; 106: 13-22.

22. Akshay A. Shah, MSc; Killian Donovan, BM, BCh; Claire Seeley, M \& al.Risk of Infection Associated With Administration of Intravenous Iron A Systematic Review and Meta-analysis JAMA Network Open. 2021;4(11): e2133935. doi:10.1001/jamanetworkopen.2021.33935
23. E. Bisbe, L. Molto, R. Arroyo, J.\& al. Randomized trial comparing ferric carboxymaltose vs oral ferrous glycine sulphate for postoperative anaemia after total knee arthroplasty. British Journal of Anaesthesia 113 (3): 402-9 (2014) Advance Access publication 29 April 2014. doi:10.1093/bja/a.u092

24. C.O. Ramos-Pẽnafiel $\square$, C. Balderas-Delgado, Á. CabreraGarcía. Surgery and transfusion Rev Med Hosp Gen Méx. 2016;79(2):98-106.

25. Shander A, Javidroozi M, Perelman S, et al. From blood-less surgery to patient blood management. Mt Sinai J Med.2012; 79:56-65.

26. Shander A, Spence RK, Auerbach M. Can intravenous irontherapy meet the unmet needs created by the new restrictions on erythropoietic stimulating agents? Transfusion (Paris).2010;50:719-32.

27. De Robertis E, Longrois D. To streamline the guideline challenge: The European Society of Anaesthesiology policy on guidelines development.Eur J Anaesthesiol 2016; 33:794799.

28. N. M. Calle, F. H. Martínez Ana Alfonso Pierola. Implementation of a management protocol for massive bleeding reduces mortality in non-trauma patients: Results from a single centre audit .July 2016, Medicina Intensiva 40(9) DOI:10.1016/j.medin.2016.05.003

29. Gilliss BM, Looney MR, Gropper MA. Reducing noninfectious risks of blood transfusion. Anesthesiology 2011; 115:635-649.

30. Edgeworth JA, Farmer M, Sicilia A, et al. Detection of prion infection invariant Creutzfeldt-Jakob disease: a blood-based assay. Lancet 2011; 377:487-493. 